\title{
Route Lifetime Based Optimal Hop Selection in VANETs on Highway: An Analytical Viewpoint
}

\author{
Dinesh Kumar, Arzad A. Kherani, and Eitan Altman
}

INRIA B.P. 93, 2004 Route des Lucioles, 06902 Sophia Antipolis Cedex, France

\{dkumar, alam, altman\}@sophia.inria.fr

\begin{abstract}
We consider the problem of optimal next-hop selection in a route between two vehicles, for a simple scenario of Vehicular ad hoc networks (VANETs) on a highway. For a given approximation of the optimal number of hops, we seek the optimal choice of next-hop based on its speed and inter-node distances, so as to maximize the expected route lifetime. Under a Markovian assumption on the process of speed of nodes, we show that the optimal choice of speeds attempts to equalize the lifetimes of adjacent links. A monotone variation property of the speed of relay nodes under the optimal policy is proved. These properties have been confirmed with simulations. The optimal policies and their structures can assist in enhancing the performance of existing VANET routing protocols.
\end{abstract}

Keywords: Vehicular ad hoc networks, optimal routing, link lifetime.

\section{Introduction}

Vehicular Ad Hoc Networks (VANETs) [1, 2, 3, 4, tend to exhibit a drastically different behavior from the usual mobile ad hoc networks (MANETs) 6]. High speeds of vehicles, mobility constraints on a straight road and driver behavior are some factors due to which VANETs possess very different characteristics from the typical MANET models. Broadly speaking, four such characteristics are rapid topology changes, frequent fragmentation of the network, small effective network diameter and limited temporal and functional redundancy [6]. Due to this fundamental behavioral difference between MANETs and VANETs, topologybased routing protocols developed for the former cannot be directly used in the latter. Topology-based protocols are the table-driven proactive protocols and on-demand reactive protocols [7]. For example authors in [10] have shown that TORA (an on-demand protocol) is completely unsuitable for VANETs. Instead, position-based routing protocols such as LAR, DREAM or GPSR [11,12, 13] that require a-priori knowledge of vehicles' geographic location (from a GPS service) could be used for VANETs for faster route discovery and improved performance. But position-based routing protocols suffer from geographic routing failures due to presence of topology holes [14] and authors in [14] propose spatially aware routing for VANETs to overcome this drawback. However optimality of spatially aware routing has not been proved and it could be further enhanced in order to improve performance. 
A routing protocol usually has three main functions: route discovery, optimal route selection (among various candidate routes discovered) and route maintenance. Once an optimal route from a source to its destination has been discovered and selected, route maintenance must be carried out, in order to track link failures (due to movement of relay nodes) and perform route re-discovery. Route maintenance and re-discovery are expensive in signalling and computation, and hence it is desirable to choose the optimal route comprising links with maximum possible lifetimes during the optimal route selection phase. In this paper we propose an optimal route selection criteria from an analytical viewpoint, for the simple scenario of a VANET on a straight line highway. Our optimal route selection criteria consists of the optimal choice of next-hop, based on maximum route lifetime. The proposed optimal next-hop selection criteria based on maximum route lifetime is not a competitor to the optimal route selection methods in any of the existing routing protocols, but rather it can be used in conjunction with them. Our goal is not to propose a complete routing protocol along with its implementation aspects. We rather focus to gain an analytical insight into the route lifetime dynamics of a VANET by considering only a simple scenario and our observations on the structural characteristics of the optimal policies can assist in enhancing the performance of any of the existing routing protocols mentioned before or spatially aware routing in particular, for VANETs. As discussed before, optimal route selection in VANETs can be very different from that in MANETs and designing a routing protocol for VANETs can be very complex due to the rapidly changing topology and frequent link breakdowns. In our model, we introduce certain simplifying assumptions, as compared to a real life scenario, in order to gain an analytical insight into the dynamics of vehicle mobility and route lifetimes in VANETs. Without these simplifying assumptions it can be very hard to study these dynamics. For instance, a VANET in city traffic scenario can be very hard to model and our analysis does not hold good for this case. The contributions of this paper are twofold. Firstly, the heuristics and structural characteristics of the optimal hop selection policies developed in this paper can assist in better understanding the dynamics of route lifetime in VANETs. Secondly, the results can serve in enhancing the performance of existing routing protocols for VANETs.

\section{Optimization Parameters}

We consider VANETs on a straight line highway in which a vehicle can establish connectivity only with other vehicles traveling in the same direction of its motion. In other words we consider ad hoc networks formed by only those vehicles that are moving on the same side of a high way and not the opposite side. Assume vehicles (nodes) traveling on an infinitely long straight highway with $L$ lanes, moving in the same direction on either side of the highway. Each lane $i$ has an associated speed limit $s_{i}$. Assume that in a given lane, the nodes travel with a speed corresponding to the speed limit of that lane. In other words, it is assumed that all nodes move on the highway with a discrete set of speeds 
which consists of the speed limits of each lane. We follow the convention that $s_{1}<s_{2}<\ldots<s_{L}$. When a node transits to an adjacent lane due to driver's natural behavior, it now travels with the speed associated with the new lane. Now consider 2 tagged nodes, a source and a destination moving in any two (possibly same) lanes, traveling in the same direction. At time 0 , these nodes are assumed to be distance $D$ apart. If $D$ is large enough then these nodes may not be able to communicate with each other directly. Intermediate relay nodes are required for these two tagged nodes to form a VANET. However more than one options (vehicles in front of transmitting vehicle moving with identical or different speeds in the same lane or adjacent lanes, respectively) for the choice of next hop may be available. How would one decide whether to choose the vehicle in the same lane or in adjacent lanes as the next hop. In this paper we address the problem of coming up with an optimal choice of next hop (relay node) such that the associated link lifetime and hence the route lifetime, is maximized. The constraints under which this decision should be made are mentioned in detail in Section 3, but here we emphasize on the fact that making such a decision may not be as simple as it seems at first. An evident reason being that the underlying state space over which the route lifetime has to be optimized is composed of different parameters, each representing as a component parameter of the overall optimization problem. Following are the possible optimization parameters that should be considered and the motivation behind their choice is discussed in a predecessor research report [5] on this work:

1. Optimization over Number of Relay Nodes

2. Optimization over Inter-node Distances

3. Optimization over Speeds of the Intermediate Nodes

In the present work, we assume that nodes (vehicles) are equipped with a GPS receiver and we also assume that the optimal number of relay nodes and the speeds of the source and destination nodes are somehow known in advance. Avoiding relatively large values for number of relay nodes, an optimal choice on number of relay nodes can be fairly approximated from the knowledge of transmission range $R$ and position of source and destination nodes obtained from the GPS receiver. Approximate speeds of source and destination nodes can also be obtained from a GPS service. Given this information, we are interested in obtaining the optimal inter-node distances and optimal speeds of relay nodes that result in a maximum possible route lifetime.

\section{System Dynamics and Model}

\subsection{Dynamics of Individual Nodes}

The process of changing speed of any individual node due to lane change on the highway is assumed to be an independent stationary ergodic stochastic process. We are thus also implicitly assuming that the vehicles do not leave the highway. It is assumed as well that the vehicles do not change their direction of motion 
since we consider VANETs formed by only those vehicles that are traveling on the same side of highway in the same direction. In this paper, we restrict ourselves to the case where the changing speed of any node can be modeled as an irreducible aperiodic Markov process, taking a finite set of constant values $\left\{s_{1}, s_{2}, \ldots, s_{L}\right\}$. We assume that a node continues to move in lane $i$ with an associated speed $s_{i}, 1 \leq i \leq L$ for an exponential amount of time before changing its lane, or its speed equivalently. This time is exponentially distributed with rate $\mu_{i}$ and we denote that a node in lane $i$ transits to another lane $j$ with probability $P_{i, j}$ with $P_{i, i}=0$. Even though our analysis holds good for generic transition probabilities $P_{i, j}$, we assume the following natural structure on node transitions in our highway scenario: from state (or, lane) $i$, a node can transit only to the states $(i-1) \vee 1$ or $(i+1) \wedge L$. Clearly, from state 1 a node can transit only to state 2 and from state $L$ the only possible transition is to state $L-1$.

\subsection{Placement of Nodes}

We assume that node spread-out along the highway is dense in the sense that in a sufficiently small neighborhood of any point on a lane we can always find at least one node on the same lane. This is like assuming that the transmission range $R$ of a node is significantly large as compared to the distances between two successive nodes in any lane. Most of the results in this paper can be extended to the case where we assume that the existence of a node at any point on a lane is itself a stochastic process. However, since we are more interested in the structural results of optimal distances and speed selections, we will assume that this stochastic process is a constant process, i.e., there is always a node at any given point on any lane. It is also assumed that the width of the lanes on an highway is negligible when compared to the transmission range of mobile nodes along the length of highway. We call this assumption as the straight line communication assumption.

\subsection{Evolution of Inter-node Distances and Node Connectivity}

Consider any two nodes $i$ and $j$ (node $j$ is ahead of node $i$ ) moving in any two lanes with both the nodes moving in the same direction. Assume that the two nodes have speeds $v_{i}(t)$ and $v_{j}(t)$ respectively at time $t$. Since the two nodes are moving and also have their speeds changing with time due to lane change, the distance between these nodes will also vary with time. Let us denote the distance of node $j$ from node $i$ (measured in the direction of motion) at time $t$ as $d_{i j}(t)$. Assume that node $i$ is the source of transmissions meant for node $j$. We say that a direct link or single hop route exists between nodes $i$ and $j$ as long as $0 \leq d_{i j}(t) \leq R$, where $R$ is the maximum possible transmission range of a node i.e. a node can successfully transmit at any range $\leq R$.

The distance between any two adjacent nodes $i$ and $i+1$ (node $i+1$ is ahead of node $i$ ) of a route denoted simply by $d_{i}(t)$, forms a stochastic process that begins with an initial value of $d_{i}(0)=d_{i}$ and whose evolution over time, $d_{i}(t)$, depends on the initial speeds of the two nodes. We assume that two successive 
nodes $i$ and $i+1$ of a route remain connected only until when $d_{i}(t)$ takes a value outside the interval $[0, R]$ for the first time (see Figure 1 ). The convention followed is that the link between two successive nodes $i$ and $i+1$ of a route, breaks, if either, 1) node $i+1$ overtakes node $i$ in the direction of motion and the distance between node $i$ and node $i+1$ exceeds $R$ so that node $i+1$ is outside the maximum transmission range of node $i$, or, 2) node $i$ overtakes node $i+1$ in the direction of motion. This convention can be easily relaxed to incorporate the case where the link between node $i$ and $i+1$ breaks only when node $i$ overtakes node $i+1$ by a distance $R$, in the direction of motion. The results of our analysis will still hold good with this relaxed convention.

In brief, we consider nodes $i$ and $j$ to be connected if node $j$ lies within the maximum transmission range of node $i$ only in the direction of motion and not otherwise. Note that since the communication devices mounted in the vehicles operate on car battery which is recharged by the vehicle engine, battery-life of nodes is not an issue in our model. All these simplifying assumptions above and in previous Section 2, have been adopted to avoid a very complex modeling scenario, since the main focus is to get an approximate first glimpse of the underlying dynamics of mobility of nodes and route lifetimes in a VANET.

Assume $M$ relay nodes in a route between the source and its destination, with the source being the $0^{t h}$ node and the destination as the $(M+1)^{t h}$ node. Let $v_{0}$ and $v_{M+1}$ be the velocities of the source and destination nodes and let $D$ be the distance between them. For a given value of $M$, let $d_{i}, 0 \leq i \leq M$ be the distance between node $i$ and node $i+1$. We impose that $\sum_{i=0}^{M} d_{i}=D$ so that the last hop distance $d_{M}=D-\sum_{i=0}^{M-1} d_{i}$. For a non-broken route formed by nodes $0,1,2, \ldots, M+1$, we require that $0 \leq d_{i} \leq R$ and let $v_{i}, 0 \leq i \leq M+1$ be the velocity of the $i^{t h}$ node with $v_{0}$ and $v_{M+1}$ known in advance. Note that $v_{i} \mathrm{~s}$ may take any one of the set of constant values $\left\{s_{1}, \ldots, s_{L}\right\}$ and there are $L^{M}$ different possible values that the vector $\underline{v}=\left(v_{1}, \ldots, v_{M}\right)$ can take.

\section{The Problem Formulation}

In our model described in the previous section, we assume a dense vehicle traffic scenario on the highway. Due to this assumption multiple candidate routes may exist for choosing an optimal route. If multiple candidate routes are available then we want to choose the route with the maximum lifetime. We are given that there are $M+2$ nodes, indexed $0,1, \ldots, M+1$, constituting a route. Node 0 is the source node and node $M+1$ is the destination node. Now consider any two successive nodes $i$ and $i+1$ in the route, that are distance $d$ apart at time zero. Assume also that at time zero, node $i$ is in lane $k$ and node $i+1$ is in lane $l$ such that $d_{i}(0)=d, v_{i}(0)=s_{k}$ and $v_{i+1}(0)=s_{l}$. Let $T\left(d, v_{i}, v_{i+1}\right)$ be the expected time after which the link between these two nodes breaks (see Section 3). We refer to the quantity $T\left(d, v_{i}, v_{i+1}\right)$ as the link lifetime of the link between the successive nodes $i$ and $i+1$ in a route.

For a route comprised of $M+1$ links, our problem is to find an optimal inter-node distance assignment denoted by $\underline{d}^{*}=\left(d_{0}, \ldots, d_{M-1}\right)$, and an optimal 
speed assignment, denoted by $\underline{v}^{*}=\left(v_{1}, \ldots, v_{M}\right)$, to the $M$ relay nodes such that maximum route lifetime is attained. We thus seek the optimal distance vector $\underline{d}^{*}$ and speed vector $\underline{v}^{*}$ such that the least of the link lifetimes of the route is maximized. Our optimization problem is therefore the following,

$$
\underset{\underline{v}, \underline{d}}{\operatorname{Maximize}} \underset{i=0 . . M}{\operatorname{Minimum}} T\left(d_{i}, v_{i}, v_{i+1}\right) .
$$

Instead of solving the above problem directly, we can also attempt to optimize a different, parameterized, objective function. This objective function will coincide with the original one in Equation 1 when the parameter takes a special value. We state here the following theorem whose proof can be found in [5].

Theorem 1. The solution of the optimization problem in Equation 1 is identical to that of the optimization problem below as $\alpha \rightarrow \infty$.

$$
\underset{\underline{v}, \underline{d}}{\operatorname{Minimize}}\left[\sum_{j=0}^{M}\left(T\left(d_{j}, v_{j}, v_{j+1}\right)\right)^{-\alpha}\right]^{\frac{1}{\alpha}},
$$

In fact, we can say something more about the relation between the two optimization problems of Equation 1 and 2 in the following theorem.

Theorem 2. There exists a finite $\alpha^{*}$ such that the maximizers of optimization problem of Equation 1 are identical to that of Equation [ 2 for all values of $\alpha>\alpha^{*}$.

The proof of this theorem can be referred to in the research report [5]. Theorem 2 ensures that there is no discontinuity in the solution of the optimization problem of Equation 2 with respect to the solution of Equation 1 as $\alpha \rightarrow \infty$. Working with the objective function of Equation 2 in fact has an advantage that we can optimize it for some finite value of $\alpha>\alpha^{*}$ and elegantly obtain the solution to the optimization problem of Equation 1 .

\section{Determining the Expected Lifetimes}

Having done with the problem formulation, here we seek to obtain explicit expressions for the link lifetimes, to be able to explicitly define the objective function of either Equation 11 or Equation 2. We study the expected lifetime of the connection between two nodes that are $d$ distance apart at time 0 and have speeds $s_{i}$ and $s_{j}$ respectively. We use the notation that a pair of nodes $k$ and $l$ is in state $s_{i j}$ when node $k$ is in lane $i$ with associated speed $s_{i}$ and node $l$ is in lane $j$ with associated speed $s_{j}$. Here onwards, along with $T\left(d, v_{k}, v_{l}\right)$, we will also use the notation $T\left(d, s_{i j}\right)$ for the link lifetimes of any two nodes, interchangeably. With some abuse of notation we use the same notation for the state $s_{i j}$ and the relative speeds between the two nodes $s_{i j} \triangleq s_{j}-s_{i}$, interchangeably. Consider a pair of successive nodes forming a link in a route as shown in Figure 1. If the second node is within the range $R$ of the first node then using the straight line 


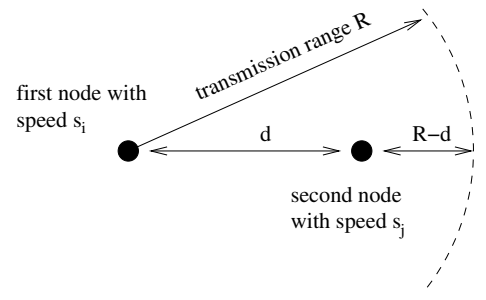

Fig. 1. Two successive nodes constituting a route path

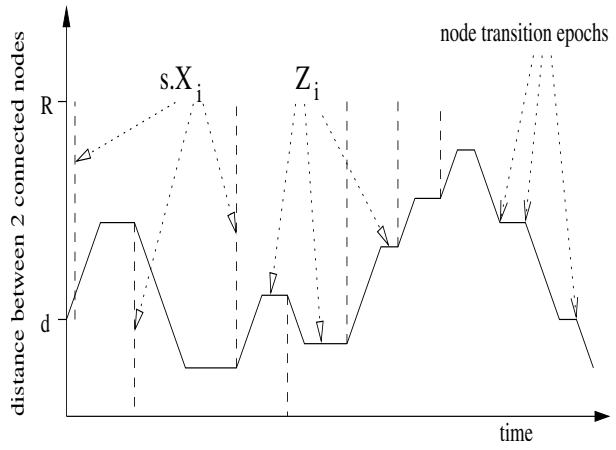

Fig. 2. Random walk model for 2 successive nodes in a route

communication assumption mentioned before in Section 3 , the expected remaining link lifetime is given by $T\left(d, s_{i j}\right)$ and we state the following theorem whose proof can be referred to in $[5]$.

Theorem 3. $T\left(d, s_{i j}\right)$ satisfies the following renewal-type recursions

$$
\begin{aligned}
s_{i j}>0 \quad T\left(d, s_{i j}\right)= & e^{-\left(\mu_{i}+\mu_{j}\right) \frac{R-d}{s_{i j}}} \frac{R-d}{s_{i j}}+\int_{0}^{\frac{R-d}{s_{i j}}}\left(\mu_{i}+\mu_{j}\right) e^{-\left(\mu_{i}+\mu_{j}\right) u}[u+ \\
& \left.\sum_{l} P_{i, l} \frac{\mu_{i}}{\mu_{i}+\mu_{j}} T\left(d+s_{i j} u, s_{l j}\right)+\sum_{l} P_{j, l} \frac{\mu_{j}}{\mu_{i}+\mu_{j}} T\left(d+s_{i j} u, s_{i l}\right)\right] d u
\end{aligned}
$$

$$
\begin{aligned}
s_{i j}<0 \quad T\left(d, s_{i j}\right)= & e^{-\left(\mu_{i}+\mu_{j}\right) \frac{d}{\left|s_{i j}\right|}} \frac{d}{\left|s_{i j}\right|}+\int_{0}^{\frac{d}{\Gamma_{i j} \mid}}\left(\mu_{i}+\mu_{j}\right) e^{-\left(\mu_{i}+\mu_{j}\right) u}[u+ \\
& \left.\sum_{l} P_{i, l} \frac{\mu_{i}}{\mu_{i}+\mu_{j}} T\left(d-\left|s_{i j}\right| u, s_{l j}\right)+\sum_{l} P_{j, l} \frac{\mu_{j}}{\mu_{i}+\mu_{j}} T\left(d-\left|s_{i j}\right| u, s_{i l}\right)\right] d u,
\end{aligned}
$$

$$
\begin{aligned}
s_{i j}=0 \quad T\left(d, s_{i j}\right)= & \int_{0}^{\infty}\left(\mu_{i}+\mu_{j}\right) e^{-\left(\mu_{i}+\mu_{j}\right) u}\left[u+\sum_{l} P_{i, l} \frac{\mu_{i}}{\mu_{i}+\mu_{j}} T\left(d, s_{l j}\right)+\right. \\
& \left.\sum_{l} P_{j, l} \frac{\mu_{j}}{\mu_{i}+\mu_{j}} T\left(d, s_{i l}\right)\right] d u .
\end{aligned}
$$

Instead of solving the system of Equations 3, 4, and 5] explicitly in its most general form, we solve it only for some special cases. The main reason for considering only these special cases is that these are the only cases which are of relevance in a real life highway scenario and solutions for cases other than these cannot be applied to real life traffic movement on highways. Another interesting aspect of considering these special cases is that the results that we obtain for these cases constitute a simple form and provide important insights into the structure of the corresponding optimal distance and speed policies. Later with the help of 
simulations we will attempt to validate the obtained structure for any general case.

In the following sub-sections we attempt to solve the link lifetime recursion equations for particular cases of $L=2$ and $L \geq 3$. The case $L=1$ is trivial because there is no breakdown of routes, since all nodes are always traveling with the same speed $s_{1}$. Firstly, we consider the case $L=2$ and, assuming $\mu_{1}=\mu_{2}$, we obtain explicit expressions for the quantities $T\left(d, s_{i j}\right)$ 's. We then solve the optimization problem of Equation 1 directly for $M=1$ and $\frac{R}{s_{12}}<\frac{1}{2 \mu}$. For values of $M>1$, the global optimization problem can be solved by splitting it into several optimization problems each one of them optimizing over a pair of two adjacent links (i.e., $M=1$ ). The solution of these split problems can then be combined to obtain solution to the global optimization problem (for $M>1$ ) after taking care of certain coupling issues related to adjacent pairs of links. Second, we consider the case with general values of $L \geq 3$ and $\frac{1}{\mu_{i}}>>\frac{R}{s_{i}}$ so that a node remains in lane $i$ for a very long period as compared to the lifetime of a link. For this case we derive only the optimal speed assignment policy, an interesting property of the optimal speed vector solution to the problem of Equation 1 and develop some structural heuristics about the optimal speed vector solution to the problem of Equation 2. Both these cases provide important guidelines on optimally choosing the inter-node distances and speed of next hop.

\section{$5.1 \quad L=2$}

Consider the case where the number of lanes is $L=2$. There are only two possible speeds $s_{1}$ and $s_{2}$ in this case with $s_{2}>s_{1}$. At any time $t$, let the source have speed $v_{0}(t)$ and destination have speed $v_{M+1}(t)$. Recall that the processes $\left\{v_{0}(t)\right\}$ and $\left\{v_{M+1}(t)\right\}$ are assumed to be independent Markov processes over the state space $\left\{s_{1}, s_{2}\right\}$. The infinitesimal generator matrix is then given by:

$$
\begin{array}{c|cc} 
& s_{1} & s_{2} \\
\hline s_{1} & -\mu_{1} & \mu_{1} \\
s_{2} & \mu_{2} & -\mu_{2}
\end{array}
$$

Here $\mu_{i}$ is the rate of the exponentially distributed sojourn time when the process $\left\{v_{0}(t)\right\}$ (or, $\left.\left\{v_{M+1}(t)\right\}\right)$ is in state $s_{i}$. We state the following lemma without proof.

Lemma 1. If $\mu_{1}=\mu_{2}=\mu$ then,

1. The process of the speed of destination node with respect to the source node, i.e., $\left\{v_{0}(t)-v_{M+1}(t)\right\}$ forms an irreducible periodic Markov process over (finite) state space $\left\{0, s_{12}, s_{21}\right\}$ with the mean sojourn time in any state being exponentially distributed with rate $2 \mu$.

2. The state transition probability matrix is of the form

\begin{tabular}{l|ccc} 
& $s_{12}$ & 0 & $s_{21}$ \\
\hline$s_{12}$ & 0 & 1 & 0 \\
0 & 0.5 & 0 & 0.5 \\
$s_{21}$ & 0 & 1 & 0
\end{tabular}


In words, from the states with non-zero relative speed, transition is always to the one with a relative speed of 0 and from the state with relative speed 0 , the transition is to either of the other two states, each with probability 0.5 .

An important consequence of the observation of Lemma 1 is that the function $T\left(d, v_{i}, v_{j}\right)$ depends on $v_{i}$ and $v_{j}$ only via $v_{i}-v_{j}$ with $v_{i}-v_{j} \in\left\{0, s_{12}, s_{21}\right\}$. We will see later that the observation of Lemma 1 also helps us to compute the function $T(d, 0)$ directly via a simple application of Wald's lemma [8, Chapter 7] without solving any integral equation for $T(d, 0)$. We have the following recursions for $T\left(d, s_{12}\right)$ and $T\left(d, s_{21}\right)$ from Equations 3 and 4 .

$$
\begin{gathered}
T\left(d, s_{12}\right)=e^{-2 \mu \frac{(R-d)}{s}} \frac{R-d}{s}+\int_{u=0}^{\frac{R-d}{s}}(u+T(d+s u, 0)) 2 \mu e^{-2 \mu u} d u, \text { for } \\
s_{12}>0, s=s_{2}-s_{1} \\
T\left(d, s_{21}\right)=e^{-2 \mu \frac{d}{s}} \frac{d}{s}+\int_{u=0}^{\frac{d}{s}}(u+T(d-s u, 0)) 2 \mu e^{-2 \mu u} d u, \text { for } s_{21}<0, s=s_{2}-s_{1}
\end{gathered}
$$

For obtaining $T(d, 0)$ we follow the approach of random walks. Recall that $T(d, 0)$ is the expected time for which the distance between the two nodes remains in the interval $[0, R]$, starting with distance $d$ apart and 0 relative speed. Clearly, the distance between the nodes can change only when the relative speed between the two nodes is non-zero. The periods of zero and non-zero relative speed alternate and the instants of the beginning of zero relative speed form renewal instants for the relative speed process.

Consider a particle starting at point $d$. As in random walks, in each time unit the particle moves to either left or right (each with probability $\frac{1}{2}$ ) and moves by an exponentially distributed amount. The mean of the jump size is $\frac{1}{m}$ where $m=2 \mu$. Let $S_{n}, n \geq 1$ be the position of particle just after $n^{t h}$ jump. It is then seen that $S_{n}=d+\sum_{i=1}^{n} X_{i}$ where $\left|X_{i}\right|$ s are exponentially distributed random variables (with rate $m$ ) corresponding to the jump sizes (see Figure2). $X_{i}$ takes negative and positive values with probability $\frac{1}{2}$ each. Let $N$ be the random variable corresponding to the number of jumps required by the particle to exit the interval $[0, R]$ with $R>d$. Let $q$ be the probability that the particle exits via $R$. The treatment of [8, Chapter 7] can then be used to show that, since $\left|X_{i}\right| \mathrm{s}$ are independent and identically distributed, $E \sum_{i=1}^{N}\left|X_{i}\right|=E[N] E\left[\left|X_{1}\right|\right]$ and $E\left[\left(S_{N}-d\right)^{2}\right]=E[N] E\left[\left|X_{1}\right|^{2}\right]$. To compute $E \sum_{i=1}^{N}\left|X_{i}\right|$, we need $E[N]$ which is derived from the second relation above as follows. Since $\left|X_{i}\right|$ are exponentially distributed, we can invoke the memoryless property of exponential distribution to see that

$$
S_{N}-d=\left\{\begin{array}{cc}
R-d+Y & \text { w.p. } q \\
-d-Y & \text { w.p. } 1-q
\end{array},\right.
$$

where $Y$ is an exponentially distributed random variable with rate $m$. Hence, $E\left[\left(S_{N}-d\right)^{2}\right]=E[N] E\left[\left|X_{1}\right|^{2}\right]=q E\left[(R-d+Y)^{2}\right]+(1-q) E\left[(d+Y)^{2}\right]$ 
$=\left(d^{2}+E\left[Y^{2}\right]+2 d E[Y]\right)+q(R-2 d)[R+2 E[Y]]$. From the above expression, since $E[Y]=E\left[X_{1}\right]=\frac{1}{m}$, we can obtain $E[N]$ if we know $q$. We now obtain $q$ using the fact that $E\left[S_{N}-d\right]=E \sum_{i=1}^{N} X_{i}=E[N] E\left[X_{1}\right]=0$ [8]. Now, using the possible values of $S_{N}-d$ mentioned in Equation $8, E\left[S_{N}-d\right]=0=$ $q(R-d+E[Y])+(1-q)(-d-E[Y])$, hence $q=\frac{d+E[Y]}{R+2 E[Y]}=\frac{m d+1}{m R+2}$ where we have used the fact that $E[Y]=\frac{1}{m}$. From this value of $q$, we get (using the fact that $E[Y]=\frac{1}{m}$ and $\left.E\left[X_{1}^{2}\right]=\frac{2}{m^{2}}\right) E[N]=\left((R-2 d)\left(d+\frac{1}{m}\right)+d^{2}+\frac{2}{m^{2}}+2 \frac{d}{m}\right) \frac{m^{2}}{2}$. Assuming $s=1$ with out loss of generality, it is then seen that $T(d, 0)=E\left[\sum_{i=1}^{N}\left(Z_{i}+\right.\right.$ $\left.\left.\left|X_{i}\right|\right)-\left(\sum_{i=1}^{N} X_{i}-(R-d)\right) I_{\left\{R-d<\sum_{i=1}^{N} X_{i}\right\}}-\left(-d-\sum_{i=1}^{N} X_{i}\right) I_{\left\{-d>\sum_{i=1}^{N} X_{i}\right\}}\right]$, where $Z_{i}$ s are also exponentially distributed random variables with rate $m$ and they correspond to the time when the distance between the two nodes does not change because of zero relative speed (see Figure 2). Using the memoryless property of exponential distribution, we see that if $I_{\left\{R-d<\sum_{i=1}^{N} X_{i}\right\}}=1$ then $\sum_{i=1}^{N} X_{i}-(R-d)$ is (independent and) exponentially distributed with rate $m$. Similarly, if $I_{\left\{-d>\sum_{i=1}^{N} X_{i}\right\}}=1$, then $\left(-d-\sum_{i=1}^{N} X_{i}\right)$ is exponentially distributed with rate $m$. Also, $E\left[I_{\left\{R-d<\sum_{i=1}^{N} X_{i}\right\}}\right]=q=1-E\left[I_{\left\{-d>\sum_{i=1}^{N} X_{i}\right\}}\right]$. Hence, $T(d, 0)=\frac{2 E[N]}{m}-\frac{1}{m}=(R-d) m d+R+\frac{1}{m}$. We can thus write explicit expressions for the link lifetimes from Equations $[$ and 7 as $T(d, 1)=m d(R-d)+2(R-d)$ and $T(d,-1)=m d(R-d)+2 d$, respectively.

\section{Optimal Speed Vector Solution to Optimization Problem of Equa-} tion 1 for the case of $\frac{R}{s}<\frac{1}{m}$. We consider the case where $\frac{R}{s}<\frac{1}{m}$. This scenario is of relevance since in normal real life highway traffic, a node remains in its lane for an average time greater than the lifetime of the link formed by this node and its next hop. Assuming $s=1$ with out loss of generality, it is easy to see that for this case $T(d, 1) \leq T(d, 0), \quad d \leq R$, and $T(d,-1) \leq T(d, 0), \quad d \leq R$. Now, let the distance between the source and destination be $D$ such that $R<D<2 R$. Thus one needs at least two hops or equivalently one intermediate relay node for communication. Let the number of intermediate relay nodes be $M=1$. Also, let the speed of destination with respect to the source be $s=1$ (i.e. $s_{i j}>0$ ). Here we find the optimal speed assignment for a fixed inter-node distance assignment and then later in the next paragraph, we optimize over inter-node distances. So for a given distance $d$ between the source and the intermediate node, the decision is to be made on the speed $v$ of the only intermediate relay node. Let the expected lifetime of the link between source and relay node be $L_{1}(v)$ and that of the link between relay node and destination be $L_{2}(v)$. The value of these quantities then are

\begin{tabular}{|c|c|c|}
\hline$v$ & $L_{1}(v)$ & $L_{2}(v)$ \\
\hline$s_{1}$ & $T(d, 0)$ & $T(D-d, 1)$ \\
\hline$s_{2}$ & $T(d, 1)$ & $T(D-d, 0)$ \\
\hline
\end{tabular}


Now, $T(D-d, 0)-T(d, 0)=m(D-R)(2 d-D)$ and $T(D-d, 1)-T(d, 1)=$ $(m(D-R)+2)(2 d-D)$. Hence, for $d>\frac{D}{2}, \arg \max _{v \in\left\{s_{1}, s_{2}\right\}}\left(L_{1}(v) \wedge L_{2}(v)\right)=s_{1}$ and for $d<\frac{D}{2}$, arg $\max _{v \in\left\{s_{1}, s_{2}\right\}}\left(L_{1}(v) \wedge L_{2}(v)\right)=s_{2}$. Thus, we see that by the solution to the optimization problem of Equation 1. for $s_{i j}>0$ the speed of the intermediate node should be the same as the speed of the farther node. Similarly, it is easy to derive that when the source node has speed $s_{2}$ and destination node has speed $s_{1}$ (i.e. $\left.s_{i j}<0\right)$ the speed of the intermediate node should be the same as the speed of the nearer node.

\section{Optimal Distance Vector Solution to Optimization Problem of Equa-} tion 1 for the case of $\frac{R}{s}<\frac{1}{m}$. As before, let the distance between the source and destination be $D$ such that $R<D<2 R$. Let the number of intermediate relay nodes be $M=1$ and without loss of generality, let the speed of destination with respect to the source be normalized with $s=1$. Then for $d>\frac{D}{2}$, it has been shown in the previous paragraph that the optimal speed selection is $s_{1}$. Now, it can be shown after simple algebra that for $T(d, 0)<T(D-d, 1)$ to hold good we must have $d>\frac{D(m(D-R)+2)-R+\frac{1}{m}}{2(m(D-R)+2)}$. Let us denote the RHS of the previous equation by $K$. Now, if $m$ is such that $K<R$ (and $\frac{D}{2}<K$ ). Then for $d<K$ we have $\min (T(d, 0), T(D-d, 1))=T(D-d, 1)$. For obtaining optimal $d^{*}$ we differentiate $T(D-d, 1)$ w.r.t. $d$ and equate it to zero, from which we get $d^{*}=D-\left(\frac{R}{2}-\frac{1}{m}\right)$. For $d>K$ we have $\min (T(d, 0), T(D-d, 1))=T(d, 0)$. For obtaining optimal $d^{*}$ we differentiate $T(d, 0)$ w.r.t. $d$ and equate it to zero to get $d^{*}=\frac{R}{2}$. For $d<\frac{D}{2}$, it has been shown in the previous paragraph that the optimal speed selection is $s_{2}$. It can be shown after simple algebra that for $T(d, 1)<T(D-d, 0)$ to hold good we must have $d>\frac{m(D-R) D+R-\frac{1}{m}}{2(m(D-R)+2)}$. Denote the RHS of the previous equation by $K^{\prime}$. Now, if $m$ is such that $K^{\prime}>D-R$ (and $\left.K^{\prime}<\frac{D}{2}\right)$ then for $d>K^{\prime}$ we have $\min (T(d, 1), T(D-d, 0))=T(d, 1)$. For obtaining optimal $d^{*}$ we differentiate $T(d, 1)$ w.r.t. $d$ and equate it to zero. We thus get $d^{*}=\frac{R}{2}-\frac{1}{m}$. For $d<K^{\prime}$ we have $\min (T(d, 1), T(D-d, 0))=T(D-d, 0)$. For obtaining optimal $d^{*}$ we differentiate $T(D-d, 0)$ w.r.t. $d$ and equate it to zero and get $d^{*}=D-\frac{R}{2}$.

\section{$5.2 \quad L \geq 3$}

\section{Some Properties of Solution to Optimization Problem of Equation 2} with $\frac{\boldsymbol{R}}{\boldsymbol{s}_{\boldsymbol{i}}}<<\frac{\mathbf{1}}{\boldsymbol{\mu}_{\boldsymbol{i}}}$. Here we derive some structural properties of the solution to the optimization problem of Equation 2 for the particular case of interest when $\frac{R}{s_{i}}<<\frac{1}{\mu_{i}}$ so that a node stays in its lane for a time much greater than its link lifetimes. Assume any value of $L \geq 3$ and consider the link lifetime dynamics of two nodes in lanes $i$ and $j$ that are separated by an initial distance $d<R$. It can be easily seen that for $i \neq j$ and $\frac{R}{s_{i}}<<\frac{1}{\mu_{i}}$, Equations 3 and 4 can be rewritten as $T\left(d, s_{i j}\right)=\frac{R-d}{s_{i j}} \forall s_{i j}>0$ and $T\left(d, s_{i j}\right)=\frac{d}{s_{i j}} \forall s_{i j}<0$. If both the nodes are initially in the same lane, then the distance between these two nodes remains constant till the instant when any one of them changes lanes, so that $\forall s_{i i}=0, T\left(d, s_{i i}\right)=\frac{1}{2 \mu_{i}}+\sum_{j \neq i} \frac{P_{i, j}}{2}\left(T\left(d, s_{i j}\right)+T\left(d, s_{j i}\right)\right)$. Now consider 
a route consisting of $M$ intermediate nodes so that the source and destination nodes have speeds $v_{0}$ and $v_{M+1}$ respectively, and let the distance vector $\underline{d}=\left(d_{0}, \ldots, d_{M}\right)$ be fixed. For obtaining the speed vector $\underline{v}=\left(v_{1}, \ldots, v_{M}\right)$ that maximizes the route lifetime, we can consider minimizing the objective function of Equation 2, Let us make a simplifying assumption here that $T(d, 0)=\infty$ so that $\frac{1}{T(d, 0)}=0$. Though this assumption is not necessary for the analysis that follows, it is well justified here for the case under consideration. We see that the objective function of Equation 2 for any given value of $\alpha$ is given by, $\left[\sum_{j=0}^{M}\left[\frac{1}{T\left(d_{j}, v_{j}, v_{j+1}\right)}\right]^{\alpha}\right]^{\frac{1}{\alpha}}$. Define $f_{i}(x, y)=\frac{1}{T\left(d_{i}, v_{i}, v_{j}\right)}$ such that $x=v_{i}$ and $y=v_{j}$. Clearly, if it is allowed to chose an intermediate node $i$ with any arbitrary continuum speed $x$ (thus not restricting to the discrete set of speeds $\left.s_{i}, 1 \leq i \leq L\right)$, the following condition should be satisfied for an optimal speed assignment to node $i, \frac{d}{d x}\left[\left(f_{i-1}\left(v_{i-1}, x\right)\right)^{\alpha}+\left(f_{i}\left(x, v_{i+1}\right)\right)^{\alpha}\right]^{\frac{1}{\alpha}}=0$. This implies, in particular, that $\frac{f_{i-1}\left(v_{i-1}, x\right)}{f_{i}\left(x, v_{i+1}\right)}=\left[-\frac{d f_{i}\left(x, v_{i+1}\right)}{d f_{i-1}\left(v_{i-1}, x\right)}\right]^{\frac{1}{\alpha-1}}$. Now it is easy to show that $\frac{d f_{i}\left(x, v_{i+1}\right)}{d f_{i-1}\left(v_{i-1}, x\right)}<0$. Taking $\alpha \rightarrow \infty$, we see that we need $\frac{f_{i-1}\left(v_{i-1}, x\right)}{f_{i}\left(x, v_{i+1}\right)}=1$, implying that the lifetimes of adjacent links should be equalized in order to optimize the objective function of Equation 2. Note that this is only a necessary condition and not a sufficient one, i.e., not all configurations that result in equal lifetimes of adjacent links will be the solution of the optimization problem under consideration. However, any solution of the optimization problem will satisfy the above mentioned property. This property also holds good for the case where the speeds of the relay nodes are restricted to a finite discrete set. However, it is obvious that exact equalization of the lifetimes of adjacent links is not achieved due to the lack of the choice of continuum set of speeds for the relay nodes. This issue and another property of monotone transition of speeds of relay nodes in an optimal policy has been discussed with detail in [5].

\section{Generic Formula for choice of Optimal Speed of Relay Nodes when}

$\frac{\boldsymbol{R}}{\boldsymbol{s}_{\boldsymbol{i}}}<<\frac{\mathbf{1}}{\boldsymbol{\mu}_{\boldsymbol{i}}}$. Here we derive a generic formula for the choice of optimal speed of a relay node (solution to the optimization problem of Equation 1) for the particular case of interest when $\frac{R}{s_{i}}<<\frac{1}{\mu_{i}}$ so that a node stays in its lane for a time much greater than its link lifetimes. Assume any value of $L \geq 3$ and consider the link lifetime dynamics of two nodes in lanes $i$ and $j$ that are separated by an initial distance $d \leq R$. As before, it can be shown that for $i \neq j$ and $\frac{R}{s_{i}}<<\frac{1}{\mu_{i}}$, Equations 3 and 4 can be rewritten as $T\left(d, s_{i j}\right)=\frac{R-d}{s_{i j}} \forall s_{i j}>0$ and $T\left(d, s_{i j}\right)=\frac{d}{s_{i j}} \forall s_{i j}<0$. Let the speed of source and destination nodes be $s_{S}$ and $s_{D}$ and for a 2-hop communication we have $M=1$. Now, if we assume continuum set of relay node speeds, then for a fixed distance vector, the relay node speeds should be such that the link lifetimes of both links are equal (as seen in the previous paragraph). Therefore if $s$ denotes the continuum speed of the relay node and $R<D<2 R$ then from $\frac{R-d}{s-s_{S}}=\frac{R-D+d}{s_{D}-s}$ we get $s=\frac{s_{D}(R-d)+s_{S}(R-D+d)}{2 R-D}$. This shows that the relay node's optimal speed is a convex combination of speeds of source and destination for a two hop route. In 
particular, at $d=R$ we have $s=s_{S}$ and at $d=D-R$ we have $s=s_{D}$. To approximate this continuum speed $s$ with one of the available discrete speeds, we take the following approach. Let $s_{i}$ be the best approximation to $s$ and let expected lifetimes of the two links be denoted by $L_{1}(v)$ and $L_{2}(v)$, where $v$ is speed of relay node.

Case $s<s_{i}$ : If $s<s_{i}$ then $s$ can either be approximated by $s_{i}$ or $s_{i-1}$. For the choice of $s_{i}$ we have $L_{1}\left(s_{i}\right)=\frac{R-d}{s_{i}-s_{S}}, L_{2}\left(s_{i}\right)=\frac{R-D+d}{s_{D}-s_{i}}$ and $L_{1}\left(s_{i}\right)<L_{2}\left(s_{i}\right)$. Similarly, we also have $L_{1}\left(s_{i-1}\right)>L_{2}\left(s_{i-1}\right)$. Therefore for $s_{i}$ to satisfy the optimality of Equation 1 we must have $L_{1}\left(s_{i}\right)>L_{2}\left(s_{i-1}\right)$ which results in the following condition on $d, d<\frac{R\left(s_{D}-s_{i-1}\right)+(D-R)\left(s_{i}-s_{S}\right)}{s_{D}-s_{i-1}+s_{i}-s_{S}}$.

Case $s>s_{i}$ : As in the previous case, with $s>s_{i}, s$ can be approximated by $s_{i+1}$ or $s_{i}$. For the choice of $s_{i+1}$ we have $L_{1}\left(s_{i+1}\right)<L_{2}\left(s_{i+1}\right)$ and for $s_{i}$ we have $L_{1}\left(s_{i}\right)>L_{2}\left(s_{i}\right)$. Now for $s_{i}$ to satisfy the optimality of Equation 11 we should have $L_{1}\left(s_{i+1}\right)<L_{2}\left(s_{i}\right)$ which gives the bound, $d>\frac{R\left(s_{D}-s_{i}\right)+(D-R)\left(s_{i+1}-s_{S}\right)}{s_{D}-s_{i}+s_{i+1}-s_{S}}$.

Combining the two aforementioned cases and generalizing for any $L \geq 3$, following is a generic formula for the choice of optimal speed of a relay node. Choose $s_{i}$ as the speed of the intermediate node, if $d \in\left[\frac{R\left(s_{D}-s_{i}\right)+(D-R)\left(s_{(i+1) \wedge L}-s_{S}\right)}{s_{D}-s_{i}+s_{(i+1) \wedge L}-s_{S}}, \frac{R\left(s_{D}-s_{(i-1) \vee 1}\right)+(D-R)\left(s_{i}-s_{S}\right)}{s_{D}-s_{(i-1) \vee 1}+s_{i}-s_{S}}\right]$. Note that here $s_{S}<s_{D}$ and $s_{S}$ and $s_{D}$ can take any values from $s_{1}, \ldots, s_{L}$. For $M=1$, if we assume continuum set of intermediate node speeds as before, then for a fixed distance vector, the intermediate node speeds should be such that the link lifetimes of both links are equal (as seen in the previous paragraph). This implies (it can be shown after some algebra) that the link lifetimes are independent of the choice of inter-node distances, thus implying a non-unique solution for the choice of relay node speeds.

\section{Simulation Study of a VANET}

In order to validate the analysis, we have developed a simulator for a VANET. With this simulator we study and validate only the structural characteristics of the optimal speed assignment policies assuming a fixed inter-node distance assignment. Due to the limitations of this simulator, we do not study the optimal inter-node distance solution. The simulator is based on the model and assumptions proposed in Section 3 and is implemented such that the nodes move in their lanes in a discrete time space. A node in lane $i$ transits to any of the adjacent lanes at the beginning of a time slot of length 0.1 seconds and the transition takes place with probability $1-p_{i}$. Given that a node in lane $i$ transits, the transition is to lane $j$ with the same lane transition probability $P_{i, j}$. For our simulations we consider the probabilities $p_{1}=\cdots=p_{L}=p$ to be identical for all the lanes. The probability $p$ is related to $\mu_{i}$ by the relation $\frac{1}{1-p}=\frac{0.1}{\mu_{i}}$ and for $\frac{R}{s_{i}}<<\frac{1}{\mu_{i}}$, it is equivalently said that $p \rightarrow 1$. The simulator computes the expected link lifetimes of all possible links by exhaustively simulating over all possible speed assignments $\underline{v}$ of the intermediate nodes for a given scenario of $M$ 
intermediate nodes, $L$ lanes, the inter-node distance vector $\underline{d}$, speeds of source and destination $v_{0}$ and $v_{M+1}$, transmission range $R$, source and destination separation $D$ and the probability $p$. Once an exhaustive set of link lifetimes for all possible values of $\underline{v}$ is obtained by employing this brut-force method, either of the objective functions of Equation 1 or 2 is applied over this set to obtain an optimal speed assignment policy.

\subsection{Simulation Scenarios}

A car battery operated mobile device has a typical transmission range of around 200 meters. We therefore consider the possible space of inter-node distances in a VANET to vary from 140 to 200 meters and transmission range of 200 meters is considered for all the simulation scenarios. It has been shown in a previous work 9] that large number of hops in an ad hoc network can significantly degrade the TCP throughput performance. Based on this result, we consider the number of hops $(M+1)$ to vary from 2 to 7 only and the distance between the source and destination nodes is varied from 800 to 1200 meters. We perform simulations for the number of lanes $L$ varying from 2 to 6 and unless explicitly stated in the discussion on the simulation results, the associated speeds are taken as shown in the table that follows,

\begin{tabular}{|c|c|c|c|c|c|c|}
\hline$l$ & 1 & 2 & 3 & 4 & 5 & 6 \\
\hline$s_{l}(\mathrm{~m} / \mathrm{s})$ & 14 & 17 & 22 & 30 & 42 & 55 \\
\hline$\approx s_{l}(\mathrm{~km} / \mathrm{hr})$ & 50 & 60 & 80 & 110 & 150 & 200 \\
\hline
\end{tabular}

In the following part of this section we discuss some of the scenarios that were simulated and compare their results with the structural results obtained analytically. A more comprehensive simulation study can be found in [5].

1. Structure of Optimal Policy for $L=2$ (Section 5.1): Figure 3 shows plots of optimal policies obtained from Equation 1 for $L=2, M=1, p=0.9995$, $D=300 m$ and $\underline{d}=(158,142)$. The figure clearly illustrates that under optimality, an intermediate node is assigned the speed of the farther node for $s_{i j}>0$ and that of the nearer node for $s_{i j}<0$.

2. Lifetime Equalization over Continuum set of Speeds for $L \geq 3$ and $\frac{R}{s_{i}}<<$ $\frac{1}{\mu_{i}}$ (Section 5.2): In Figure 4 we consider the scenario $L=3, M=1$, $v_{0}=s_{3}=22 \mathrm{~m} / \mathrm{s}, v_{2}=s_{1}=14 \mathrm{~m} / \mathrm{s}, p=0.99999, D=300 \mathrm{~m}$ and $\underline{d}=$ $(143,157)$. In order to be able to validate the equalizing structure obtained in Section 5.2 over a continuum set of intermediate node speeds, we vary the speed associated with lane 2 from $14 \mathrm{~m} / \mathrm{s}$ to $30 \mathrm{~m} / \mathrm{s}$ in small steps of $1 \mathrm{~m} / \mathrm{s}$ and plot the link lifetimes for each such speed of lane 2 separately. This allows the only intermediate node 1 to be assigned one of the quasicontinuum set of speeds for the optimization problem of Equation 2, It is seen in the figure that under optimality, for varying values of $v_{1}$, the optimal lifetimes of the links between node 0 and 1 and node 1 and 2 are different. However at $v_{1}=23 \mathrm{~m} / \mathrm{s}$ the optimal lifetimes of the two adjacent links are almost equal thus confirming our result obtained in Section 5.2 that 

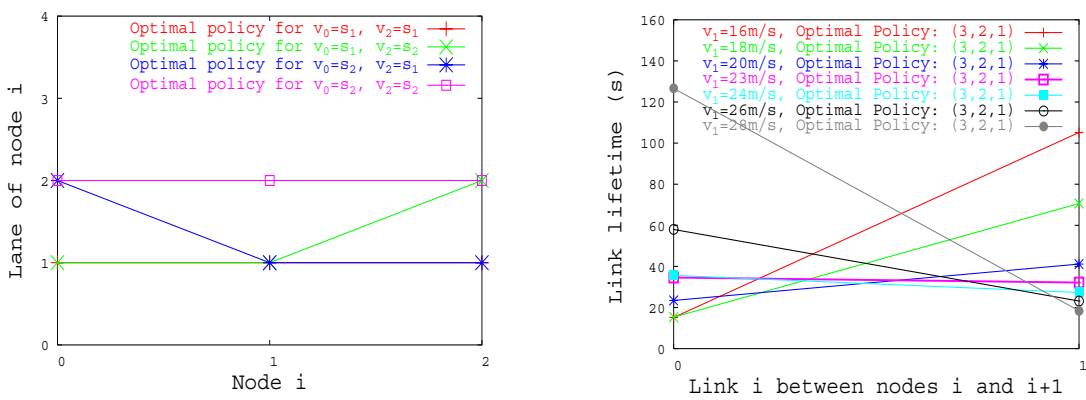

Fig. 3. Structure of optimal policy for Fig. 4. Lifetime equalization over continuum $L=2$ of speeds

the lifetimes of adjacent links should be equalized in order to optimize the objective function of Equation 2. In fact, it can be observed that we obtain the maximum of the least of the two lifetimes for speed $v_{1}=23 \mathrm{~m} / \mathrm{s}$ and the optimal lifetimes obtained for other values of $v_{1}$ are not truly optimal because of the unavailability of the choice of speed $23 \mathrm{~m} / \mathrm{s}$ in those scenarios.

\section{Conclusion}

Designing efficient routing protocols for VANETs is quite a challenging task owing to the fast speed of nodes and mobility constraints on the movement of nodes. An attempt has been made in this paper to help accomplish this task better. Under some simplifying assumptions, the analysis of this paper has established that the solution of the optimization problem under consideration tends to equalize the lifetimes of adjacent links in a route. Moreover, there is a monotone variation of the speeds of intermediate relay nodes under the optimal policy. These solution structures have also been confirmed with simulations. The structures obtained are of considerable practical interest as they reduce the space over which an existing VANET routing algorithm would search for the optimal routing policy.

\section{References}

1. J. Luo, J.P. Hubaux. "A Survey of Inter-Vehicle Communication", Tech. Rep., EPFL, Switzerland, '04.

2. Safe and comfortable driving based upon inter-vehicle communication, "w3.cartalk2000.net".

3. Car to car communication consortium, "w3.car-to-car.org". OverDRiVE project, "w3.ist-overdrive.org".

4. PReVENT: A European program to improve active safety, "w3.prevent-ip.org".

5. D. Kumar, A. A. Kherani, E. Altman. "Route Lifetime based Interactive Routing in Intervehicle Mobile Ad Hoc Networks", Research Report, INRIA, France, Sept2005. w3.inria.fr/rrrt/rr-5691.html. 
6. J.J. Blum, A. Eskandarian and L.J. Hoffman. "Challenges of Intervehicle Ad Hoc Networks", IEEE Transactions on Intelligent Transportation Systems, Vol. 5, No. 4, Dec 2004.

7. Royer et al., "A review of current routing protocols for ad hoc mobile wireless networks", IEEE Personal Communications, Apr'99.

8. R. G. Gallager. "Discrete Stochastic Processes", Kluwer, 1998.

9. Gerla et al., "TCP Performance in Wireless Multihop networks", IEEE WMCSA, Feb'99.

10. S. Jaap, M. Bechler, L. Wolf. "Evaluation of routing protocols for vehicular ad hoc networks in city traffic scenarios", 11th EUNICE Open European Summer School on Networked Applications, Spain, July'05.

11. Y.B. Ko, N.H. Vaidya. "Location-aided routing in mobile ad hoc netoworks", MobiCom'98, Oct'98.

12. Basagni et al., "A distance routing effect algorithm for mobility (DREAM)", MobiCom'98, Oct'98.

13. Karp et al., "GPSR: Greedy Perimeter Stateless Routing for Wireless Networks", MobiCom 2000.

14. Jing Tian, Lu Han, Kurt Rothermel. "Spatially Aware Packet Routing for Mobile Ad Hoc Inter-Vehicle Radio Networks", IEEE ITSC, Shanghai, China, October $12-15,2003$. 